\title{
Validating a Novel Conflict Resolution Strategy Selection Method (ConfRSSM) Via Multi-Agent Simulation
}

\author{
Alicia Y.C. Tang \\ College of Computer Science and Information Technology \\ Universiti Tenaga Nasional \\ 43000 Kajang Selangor Malaysia
}

\author{
Ghusoon Salim Basheer \\ College of Graduate Studies \\ Universiti Tenaga Nasional \\ 43000 Kajang Selangor Malaysia
}

\begin{abstract}
Selecting a suitable conflict resolution strategy when conflicts appear in multi-agent environments is a hard problem. There is a need to develop a method that can select a suitable strategy which guaranties low cost in terms of the number of messages and time ticks. This paper focuses on conflicts over agents' individual opinion and decision making by taking into account an agent's features such as collaborative, autonomous, and local communication. The significance of this research is two-fold. Firstly, this research attempts to prove the significance of giving agents the ability to select an appropriate strategy in different conflict states depending on conflict specifications such as conflict strengths and confidence levels of the conflicting agents. Secondly, the study developed a new method named as ConfRSSM for reducing the communication cost and time taken for selecting a conflict resolution strategy. The approach ignores some conflict states, and replaces complex strategies by a simpler one, in some conflicting cases. Results show ConfRSSM reduces the number of messages and time ticks and thus improving the entire conflict resolution process.
\end{abstract}

Keywords-Multi-agent, conflict resolution strategy; conflict states; confidence level; simulation

\section{INTRODUCTION}

In Multi-Agent Systems (MAS), conflicts occur when two agents have dissenting opinions on the same subject [1]. The general model for resolving a conflict is either by avoiding or solving it by using conflict resolution algorithms, or negotiation protocols [2]. In distributed, dynamic and complex environments, conflict resolution is often essential because of computational and communication bottleneck, as a result, conflict resolution is a huge challenge in multi-agent systems, and agents need to resolve conflicts in a distributed manner without global knowledge [2]-[4]. In MAS, conflict considered as a failure or a synchronization problem [4]. Choosing the most appropriate conflict resolving approach ensures proper operation of the multi-agent system. The capability of strategy selection can enhance MAS's flexibility and adaptability to dynamic and uncertain environments [5]. A significant challenge in the research on agent's conflict is the question of how to select an appropriate conflict resolution strategy.

Indeed, there is no one strategy that works best for all situations [6]. Some conflict states can be solved without using complex computational strategies such as negotiation. Strategies such as ignoring, submitting or forcing that need less computational complexity are sufficient. For this reason, developing methods for choosing among conflict resolution strategies is considered an important matter. Existing work on conflict resolution suffers from the following deficiencies:

- No technique available for detecting the confidence level of conflicting agents that takes in consideration three integrated factors, trust, certainty, and evidences. Some research builds a system that detects evidence depending on past experience [7], while other researches exploit the relation between evidence and certainty [8]. Some of the work associate certainty with the number of collected evidence. Some research evaluates trustworthiness depends on two sources of information: direct trust evidence and third party witness [9], while others build systems that detect evidence depending on the reputation of the agents [10]. It is argued that there is no formal technique for detecting agents' confidence levels that integrates trust, certainty and evidences.

- Researchers did not provide any model to detect conflict strengths and conflict classification. Conflict classification allows for identification and design of different methods for resolving conflict. Some research classified conflicts into two types: Potential conflict and real conflict [11], while others classified conflicts into two main classes: Physical conflicts and knowledge conflicts [2]. There is no model to detect the strength of an agent's conflict.

- Researchers have not discussed the relationship between conflict specifications and conflict resolution strategy selections. They did not provide a method to select a suitable conflict resolution strategy that solves conflicts among agents in all conflict states.

- In learning style detecting field, research only considers learners' responses to a specific questionnaire and detects learning styles from learner's behaviors and actions. These systems do not exploit other information such as the learners' social surrounding to detect learning styles. There is no model for learning style detection that considers the opinions of student's social surrounding. 
The paper is organized as follows: Section II provides the background of this work with the research problems and objectives. Section III presents the research methods. Section IV discusses the ConfRSSM simulation. Result discussion is presented in Section V, and Section VI concludes the paper.

\section{BACKGROUND}

\section{A. Previous Work}

Belief-Desire-Intention (BDI) agents typically have various goals they are tracking of simultaneously. In some states, the goals are inconsistent, choices made about how to pursue each of these goals may well result in a set of conflicting intentions. Conflict Resolution (CR) is the fundamental process for coordinated agent attitude. Conflict resolution includes conflict detection that involves searching for solutions, and reaching an agreement through communication among agents [12]. The capability of strategy selection can enhance MAS's flexibility and adaptability to dynamic and uncertain environments. There are several issues that must be addressed to achieve this goal. A uniform representation of a different strategy is for the comparison and evaluation processing. A metal-level reasoning mechanism for strategic decision making, a set of specifications involving requirements for a domain that agents use to evaluate substantial strategies, and the ability for adaptation to improve the decision making required to select a strategy [5].

Adler et al. [13] allowed an agent to select a specific strategy from many other strategies such as priority agreement, negotiation, arbitration, and self-modification. In their work, if there is heavy network traffic, an agent selects the arbitration strategy to resolve conflict, but if there is light traffic, the agent selects negotiation or another strategy. Liu et al. [3] mentioned the importance of allowing agents to select an appropriate conflict resolution strategy based on many factors such as conflict's nature (if there is a conflict in goal, plan or belief), the agent's autonomy level, and the agent's solution preferences.

This research provides the main framework that comprised of Agent Confidence Detection Technique (AgConfDT) that detects agent's confidence levels, and a Conflict Strength Detection Model (CSDM) that detects conflict strengths. This information is used by a Conflict Resolution Strategy Selection Method (ConfRSSM) for selecting a suitable conflict resolution strategy. Then a new model for learning style detection is used for system validation and evaluation. AgConfDT includes an exploration of the three different confidence factors (trust, certainty, and evidences). It emphasizes important objects by integrating these factors in order to better understand the agents' specifications since the technique can detect the agent's confidence in the absence of any required information. Results show that the proposed technique eliminates untested opinions, such that the confidence levels of conflicting agents can be detected in all cases although in the absence of some confidence factors. CSDM detects the disagreement degree among the conflicting agents, a conflict ratio as input for the model, and the output is the conflict strength. In resolving a conflict, ConfRSSM uses the confidence levels of conflicting agents and a conflict strength to select a suitable strategy.

Finally, we propose a new model for learning style detection. The model detects students' learning styles depending on social surrounding's opinions. The run-time model enables us to evaluate the strategy performance in various computing and networking environments. Simulation results show that the proposed model provides more accurate detection of a student's learning style. This part forms the basis of the discussion of this paper.

In a Learning Management System (LMS), individuals have different learning preferences that help them learn better. These preferences are named learning styles. Many educational theorists and researchers consider learning style as an important factor that affects the learning process. Recently, more attention has given to the use of multi-agent systems in many distributed applications. Studies in multi-agent systems include the inquiry for rational, autonomous and flexible behavior of entities, and their interaction and coordination in different areas [14]. The foundation of multi-agent systems play a significant role in the growth of teaching systems, because the basic issues of teaching and learning could be easily resolved by multi-agent systems [15].

\section{B. Research Problems and Objectives}

The objectives of this work are:

a) To propose an integrated model for detecting agents' confidence levels that considers certainty, trust, and environmental evidence [16].

b) To propose a model for detecting the conflict's strength that considers the number of conflicting agents and conflicting issues in conflict states [17]-[19].

c) To propose a new model for detecting learner's learning style that considers learner's social surrounding opinions; for validating the entire framework [20], [21].

d) To formulate a novel selection strategy method for a conflict resolution in multi agent systems [24].

e) To validate (d) using agent-based simulation.

Items (d) and (e) are the focus of this paper.

\section{RESEARCH METHOD}

The research focuses on the formalization of a three frameworks: agent confidence detection, conflict resolution strategy selection, and learning style detection. The confidence detection model starts with identifying three factors that involve agent trust, agent certainty, and an evidence. The findings are used in modeling conflict resolution strategy selection. Learning style detection was selected as the domain for validating the framework. Besides analyzing common dimensions when detecting learning styles, social surrounding opinions to deliberate the detection of learning styles were added to the model. This component solicits information from parents and teachers. Conflicts may occur due to these opinions. The outcome of the study is a conflict resolution strategy selection framework that addresses agent confident, and conflict strength to select the most suitable conflict resolution strategy, as shown in Fig. 1. 


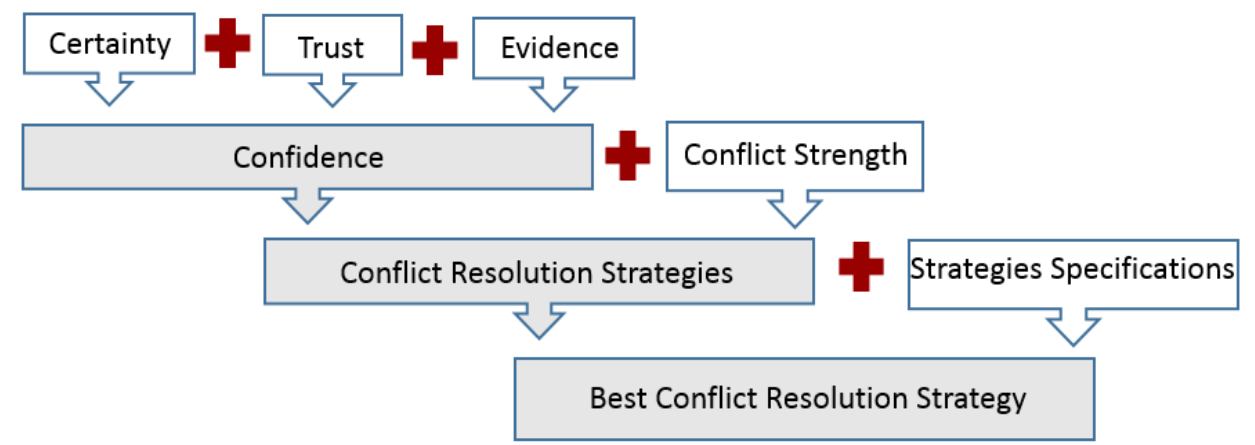

Fig. 1. The proposed conflict resolution strategy selection framework.

\begin{tabular}{|c|c|c|c|}
\hline Literature Review & \multicolumn{2}{|c|}{ Developing Prototype } & Testing \\
\hline Problem Formalization & Designing Models & Experiments & \\
\hline Conflict Resolution Strategies & $\begin{array}{c}\text { Conflict Resolution } \\
\text { Strategy Selection Model }\end{array}$ & & Assessment \\
\hline Conflict Resolution Strategies Selection & $\begin{array}{l}\text { - Environment } \\
\text { - Requirements }\end{array}$ & & cation \\
\hline Conflict Classification & $\begin{array}{c}\text { Conflict Classification } \\
\text { Model }\end{array}$ & & $\begin{array}{l}\text { - Performance } \\
\text { - Utility }\end{array}$ \\
\hline Agents' Confidence level Detection & $\begin{array}{l}\text { Agent's Confidence } \\
\text { Detection Model }\end{array}$ & & \\
\hline Trust, Certainty, Reputation & $\begin{array}{l}\text { Reputation } \\
\cdot \quad \text { Confidence }\end{array}$ & & Conclusion \\
\hline Evidences & - Integration of & & \\
\hline Learning Style detection Methods & & Implementation & \\
\hline Learning Style Detection Models & earning & Learning Style Detection System & \\
\hline Curry Onion Model & - Assessment technique & Assessment Technique & - Suggestions \\
\hline
\end{tabular}

Fig. 2. The entire research framework.

The entire framework is shown in Fig. 2. This paper presents and discusses the prototype development with the simulation results (middle column of Fig. 2). Learning style detection was used as the platform to validate the confidence model and conflict resolution selection strategy method.

\section{A. Implementation Essentials}

On the LMS part, most systems only considered learners' responses to a specific questionnaire and detect learning styles from learner's behaviors and actions. These systems do not exploit other information such as the learners' social surrounding to detect learning styles. The proposed method involves collecting four different opinions, three opinions from the student's social surrounding, (parent, teacher, and friend). The fourth opinion is collected from the student agent. VARK model questionnaires [22], [23] that deal with multiple students' personal activities and behaviors were also distributed.

\section{B. Data Gathering}

Data collection involves identifying a group of students and their social surrounding (parents, teachers, and friends). Students are required to attempt the VARK questionnaire, and their social surroundings are required to attempt different questionnaires.

\section{Testing}

To test the proposed ConfRSSM and AgnConfD models, the visual environments were created. Each visual environment represents a learning style detection scenario, which include agents that cooperate and achieve tasks that involve numerous parameters and settings. The process starts by creating four 
agents in Matlab, first agent represents a student, while other three agents represent his/her social surroundings, each of these four agents use different questionnaires for detecting a student's learning style. To simulate agents' confidence level detection, each agent uses the developed questionnaires for detecting a student's learning style. After detection, an evaluation agent will collect all agents' opinions. The evaluation agent detects conflict states and a conflict strength for each state. Based on the AgnConfD technique, the confidence level of an agent is calculated. To simulate ConfRSSM, conflicts appeared in the first simulation were used. From here, conflict strength can be detected.

\section{CONFRSSM SIMULATION}

\section{A. Simulation Environment}

The simulation is presented as a scenario of agents to select conflict resolution strategy by exploiting the ConfRSSM. The scenario includes four agents (student, parent, friend, and lecturer agents) in a learning management system. Experiments were conducted to explain how conflict states among agents are resolved, and how the conflict resolution strategy is selected based on the confidence level of conflicting agents. Many tests were generated to show the different in the number of messages and time ticks that are needed for resolving many types of conflicts.

Two experiments were conducted. The first experiment attempts to resolve conflicts by using a unique strategy (Negotiation and Arbitration). In the second experiment, the conflict resolutions are equipped with ConfRSSM. Multiple tests were run to explore the effects of environmental setting on the success of conflict resolution by a minimum number of messages and time ticks. The interface window shown in Fig. 3 is used to collect agents' opinions and calculate the conflict strengths among them. The GUI consists of two buttons, the first one collects agents' opinions, while the second button detects conflict strengths. Fig. 4 shows the results of collecting four agents' opinions and the detected conflict strengths.

An interface window (Fig. 5) was created to calculate and display the number of messages and time ticks needed for resolving conflicts among agents by using Negotiation and Arbitration strategies. The GUI consists of three columns: the first one receives the number of conflicting agents, number of proposals, and a CR strategy. The second and third columns display the number of messages and time ticks needed for resolving conflicts using the selected strategy. Fig. 6 receives the confidence values of the evaluation agents, detects and displays a suitable conflict resolution strategy for each conflict state. The GUI consists of five columns: the first one receives the agents' confidence levels from user, while the second column displays the conflict strength for each conflict state. The third column displays the conflict resolution strategy for each conflict state. The fourth and fifth columns display the number of messages and time ticks needed for each conflict resolution operation.

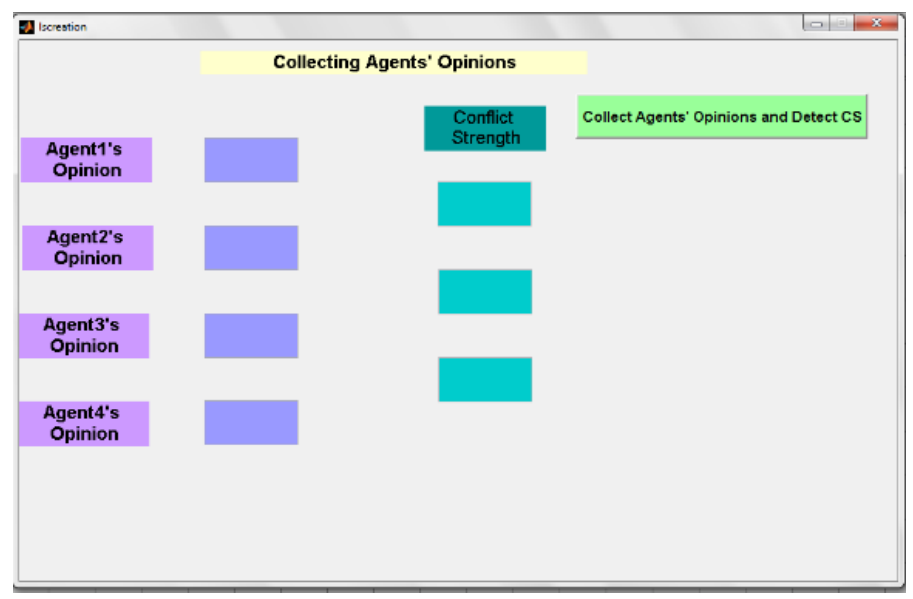

Fig. 3. The interface that collects agents' opinions and detects conflict strength.

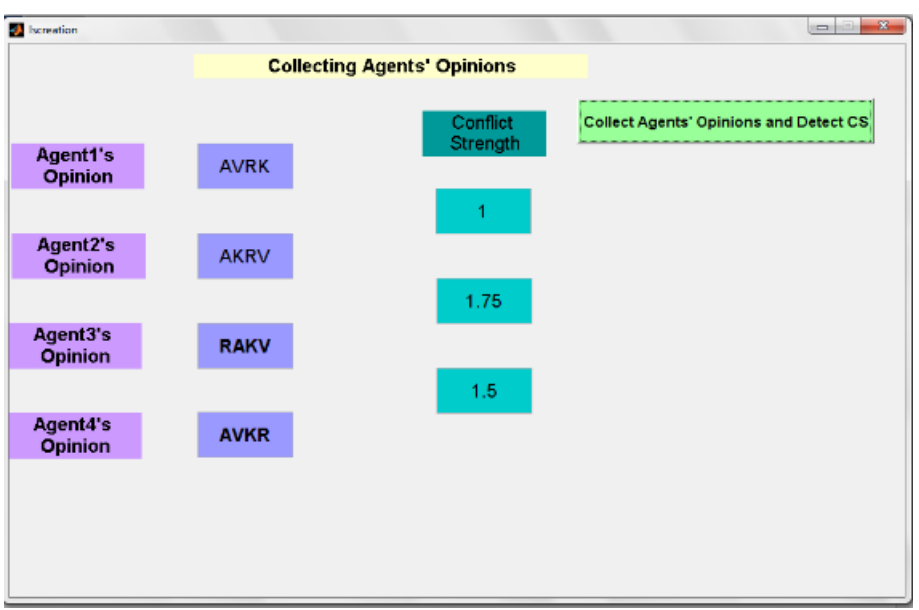

Fig. 4. The interface shows the collected agents' opinions and the detected conflict strength.

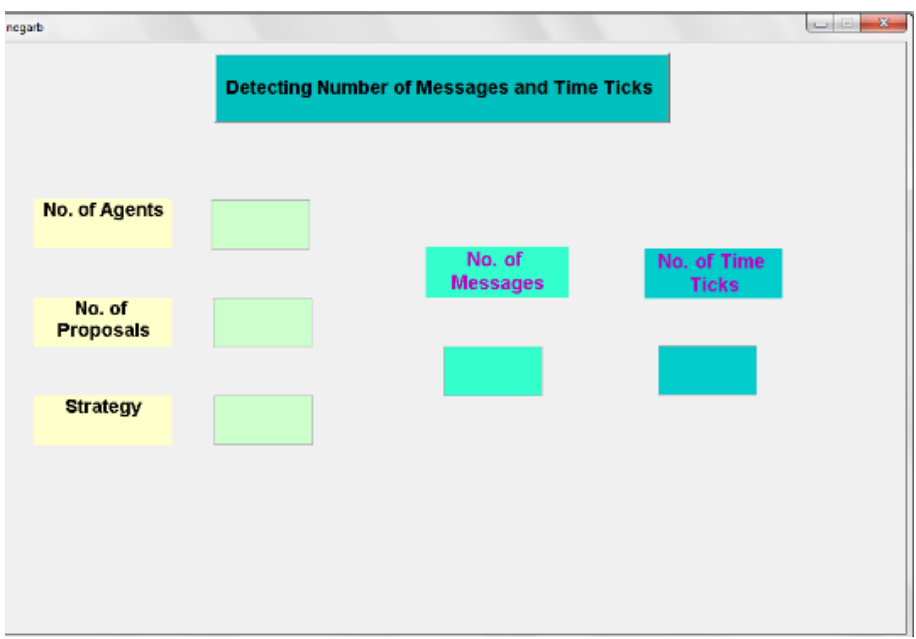

Fig. 5. The interface to calculate the number of messages and time ticks for CR strategies. 


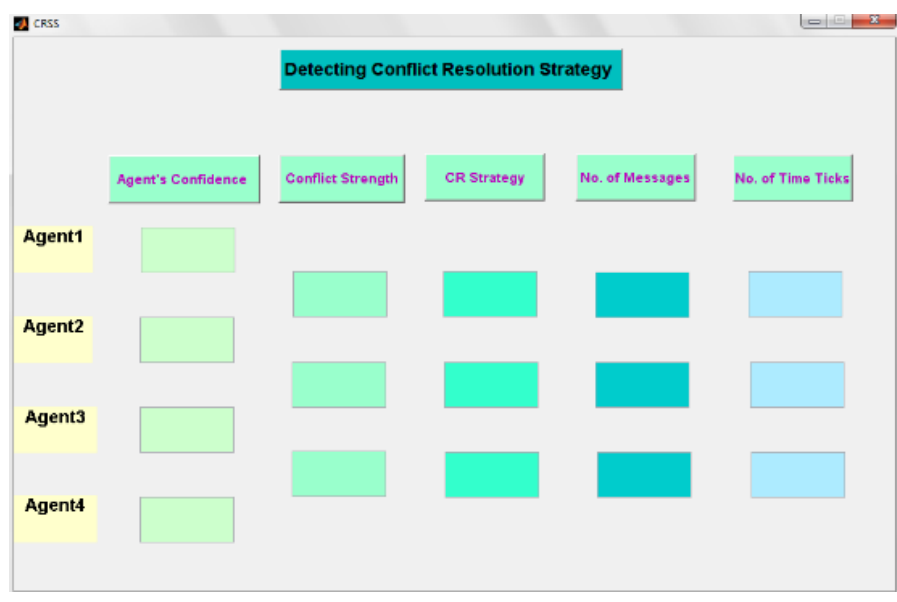

Fig. 6. Main simulation interface to run ConfRSSM.

\section{B. Observing the Number of Conflicting Agents}

Conflict states include conflicts between two agents, and conflicts among three or four agents. In the learning style detection scenario, four agents are used, a Conflicting Agent Set, CAS, is defined as a set of pairs of conflicting agents, i.e., if ai conflicts with aj, then $\mathrm{CAS}=\{(\mathrm{ai}, \mathrm{aj})\}$. Assuming that the four agents have varying levels of confidence, the following cases are apparent:

Case 1: When a conflict occurs between two agents, $\left(a_{i}\right.$, $\left.a_{\mathrm{j}}\right) \in \mathrm{CAS}$, both of them have High Level of Confidence (HLC), Confa $\mathrm{i}_{\mathrm{i}}=$ Conf $_{\mathrm{j}}$.

Case 2: When a conflict occurs between two agents, (ai, aj) $\in$ CAS, both of them have Low Level of Confidence (LLC), Confa $_{\mathrm{i}}=$ Conf $_{\mathrm{j}}$.

Case 3: When a conflict occurs between two agents, (ai, aj) $\in$ CAS, one of them has High Level of Confidence (HLC) and other have Low Level of Confidence (LLC), Confa $a_{i}>$ $\operatorname{Conf}_{\mathrm{j}}$ or or $\mathrm{Confa}_{\mathrm{i}}<\operatorname{Conf}_{\mathrm{j}}$.

In the simulation, there is a conflict resolution strategy selection agent ass that is responsible for the selection of an appropriate conflict resolution strategy in each conflict state.

\section{Variables Setting}

Each variable is defined as follows:

Conflict Strength: If there is a conflicting agent set (CAS); that conflicts about specific issues I, each conflict state has a strength of conflict, weak conflict or strong conflict. For each pair of conflicting agents $\left(a_{i}, a_{j}\right) \in C A S$, their conflict strength is represented by $\mathrm{CS}_{\mathrm{ij}}$.

Determining the Conflict Strength (CS) among Conflicting Agents: Each agent of the conflicting agents has a specific opinion about a student's learning style. The detected learning style of the student could be VARK, KVAR, and ARKV ... etc., each two conflicting agents are conflicts about the number of style (issues).

Determining the Dissenting Issues: There are issues which serve as "conflicts" among the agents. It is defined as the ratio of the number of dissenting issues to the total number of issues in one conflicting state.

$$
D=\frac{\text { Number of Dissenting Issues }}{\text { Total Number of Issues }}
$$

Determining the Conflict Ratio: Defined as a ratio of the number of conflicting agents to the total number of agents in a one conflicting set.

$$
C R=\frac{\text { Number of Conflicting Agents }}{\text { Total Number of Agents }}
$$

Learning Style Generator: This generates learning style for each agent $\left(a_{S}, a_{\mathrm{P}}, a_{\mathrm{F}}\right.$ and $\left.\mathrm{a}_{\mathrm{T}}\right)$. The generator uses a random function to produce a learning style (LS). Example: for $\mathrm{a}_{\mathrm{S}}$ as KVRA, for $\mathrm{a}_{\mathrm{P}}$ as RKVA, and for $\mathrm{a}_{\mathrm{T}}$ as VKAR.

The Domain Style (VARK): This defines the patterns of the learning style domain and their four levels:

- High level mode (HLM): The first style in the detected learning style.

- First moderate level mode 1 (MLM1): The second style in the detected learning style.

- Second moderate level mode 2 (MLM2): The third style in the detected learning style.

- Low level mode (LLM): The fourth style in the detected learning style.

Calculating the Dissenting Degree: For each conflict state, the dissenting degree is calculated using the formula: if TI is a number of a total issues in the system, and $i$ is the number of issues that agents are conflicting about it, then,

$$
\mathrm{DD}=\mathrm{i} / \mathrm{TI}
$$

Calculating the Conflict Ratio: For each conflict state, the conflict ratio is High value if more than $50 \%$ of agents in the system conflict with the rest of the agents.

Calculating the Conflict Strength (CS): For each conflict state, the CS is calculated using the formula:

$$
\mathrm{CS}=\mu \mathrm{CR}+\mu \mathrm{DD}
$$

Calculating the Number of Messages for each Strategy: For each test, the number of messages is calculated after selecting suitable strategies that resolved the conflict. Five strategies are available in the ConfRSSM method: Negotiation, Arbitration, Ignoring, Submitting, and Forcing.

\section{Experiments}

1) Test Cases without ConfRSSM

Experiment 1: When the conflicts resolution is not equipped with ConfRSSM. Conflict resolution strategies used: Negotiation and Arbitration.

Test 1: This test measures the number of messages that are needed for conflicts resolution among different number of agents, with two, three, four and five proposals, when the conflict resolution strategy is Negotiation.

Fig. 7 shows the number of messages for Negotiation. All conflicts are resolved by a unique strategy (Negotiation), without any consideration to the confidence level of conflicting agents or conflict strength among them. 


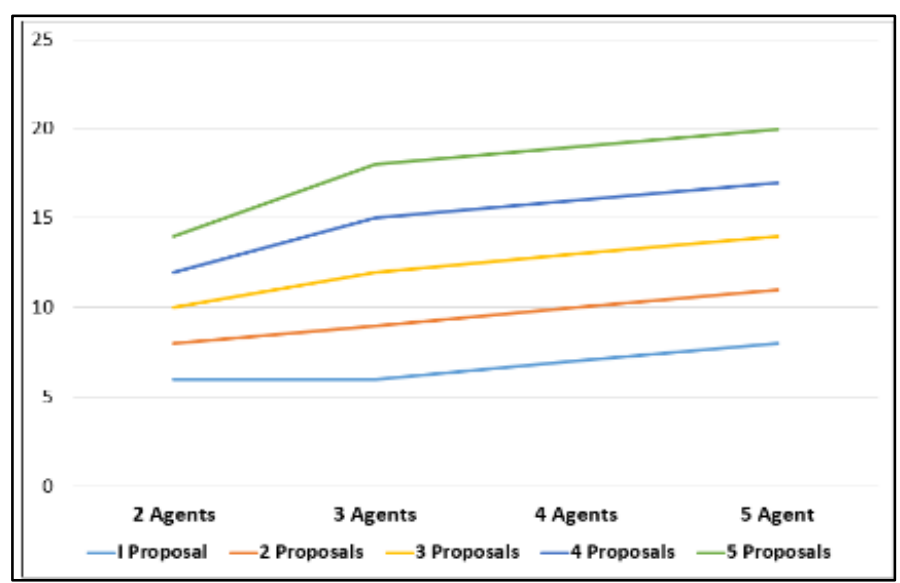

Fig. 7. The number of messages for conflict resolution using negotiation strategy.

Test 2: This test measures the number of messages needed for conflicts resolution among different number of agents, when the conflict resolution strategy is Arbitration.

Fig. 8 shows the number of messages for Arbitration. All conflicts are resolved by a unique strategy (Arbitration), without any consideration to the confidence level of conflicting agents or conflict strength among them.

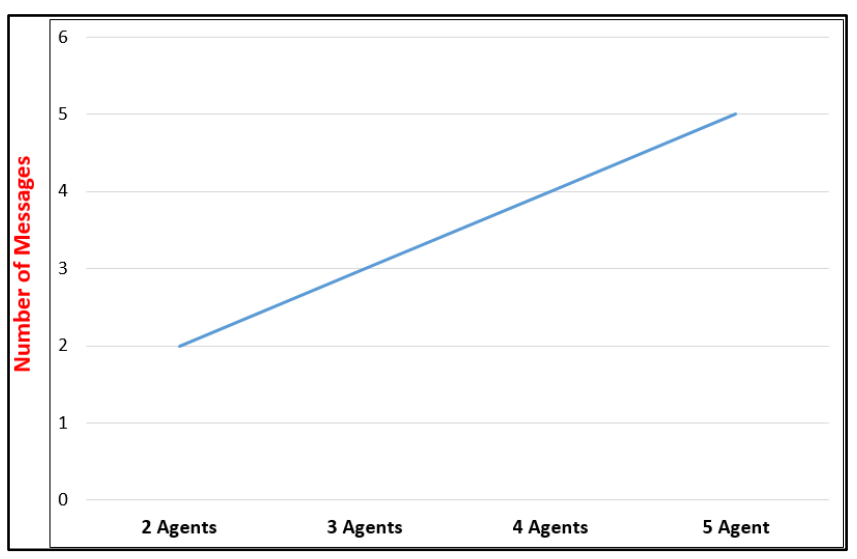

Fig. 8. The number of messages for conflict resolution using arbitration strategy.

To determine how much time is used by the Negotiation and Arbitration strategies, the outcomes on the time taken (in CPU milliseconds) for both strategies, and for each conflict states were plotted. Fig. 9 shows that the number of time ticks in three iterations increases gradually from five ticks in iteration 1 to ten ticks in iteration 2 , and fifteen ticks in iteration 3 (Negotiation strategy), and from two ticks in iteration 1 , to four ticks in iteration 2 , and to six ticks in iteration 3 (Arbitration strategy).

Discussion: Test 1 and Test 2 use the same strategy for all conflicts in all conflict states. Note that the system is unable to detect unimportant conflicts that can be ignored (or can be resolved by other strategies). If there is more than one proposal in Negotiation strategy, the number of messages increases rapidly. Clearly, as the number of proposals increase, the faster the number of messages increases as the number of agent increases.

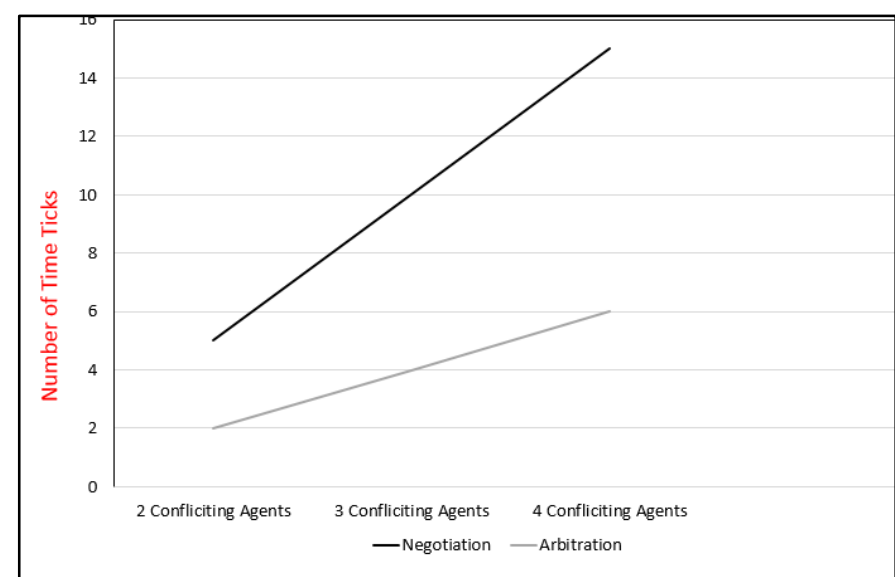

Fig. 9. The number of time ticks for resolving conflicts by using negotiation and arbitration strategies.

In Negotiation strategy, the best case is where the first proposal is accepted. On the other hand, more involved agents require more messages. It is clear that the number of messages and time ticks needed for conflict resolution among agents by using a Negotiation for more one proposal are considered high in comparison with the number of messages needed in Arbitration strategy.

In Arbitration strategy, because the same agent plays both roles, this is the only strategy that does not require inter messages, the number of messages is linear to the number of agents involved.

\section{2) Test Cases Equipped with ConfRSSM}

Experiment 2: The experiment presents an analytical model for conflict resolution that is equipped with ConfRSSM, which takes into account conflict strength and confidence level of conflicting agents. Two main factors were considered, conflict strength and confidence level of conflicting agents. Selected cases with simulation results are presented in the following sections:

Case No. 1: Weak Conflict, with five conflicting states:

- When all agents have a low-level confidence.

- When all agents have high-level confidence.

- When $50 \%$ of agents have low level confidence and $50 \%$ of agents have high-level confidence.

- When $25 \%$ of agents have low level confidence and $75 \%$ of agents have high-level confidence.

- When $25 \%$ of agents have high level confidence and $75 \%$ of agents have low-level confidence.

Case No. 2: Strong Conflict, with five conflicting states:

- When all agents have low-level confidence.

- When all agents have high-level confidence.

- When $50 \%$ of agents have low level confidence and $50 \%$ of agents have high-level confidence.

- When $25 \%$ of agents have low level confidence and $75 \%$ of agents have high-level confidence. 
- When $25 \%$ of agents have high level confidence and $55 \%$ of agents have low-level confidence.

Test 3: When all conflicting agents have a high confidence level and the conflicts among them are strong. Setting used: CAS: $a_{\mathrm{S}}, \mathrm{a}_{\mathrm{P}}, \mathrm{a}_{\mathrm{T}}, \mathrm{a}_{\mathrm{F}}$; CS: Strong; Conf $\mathrm{a}_{\mathrm{S}}$ : HCL; Conf $\mathrm{a}_{\mathrm{P}}$ HCL; Conf $\mathrm{a}_{\mathrm{T}}$ HCL; Conf $\mathrm{a}_{\mathrm{F}:}$ HCL. Table 1 shows the number of messages and time ticks for resolving the conflicts among four agents.

Test 4: When all conflicting agents have a low confidence level and the conflicts among them are strong. Setting used: CAS: $\mathrm{a}_{\mathrm{S}}, \mathrm{a}_{\mathrm{P}}, \mathrm{a}_{\mathrm{T}}, \mathrm{a}_{\mathrm{F}}$; CS: Strong; Conf as: LCL; Conf $\mathrm{a}_{\mathrm{P}}$ : LCL; Conf $\mathrm{a}_{\mathrm{T}}$ LCL; Conf $\mathrm{a}_{\mathrm{F}}$ : LCL. Results are tabulated in Table 2.

Test 5: This test detects suitable strategies for resolving strong conflicts when $50 \%$ of conflicting agents $\left(a_{S}, a_{p}\right)$ have a high level of confidence and $50 \%$ of agents $\left(\mathrm{a}_{\mathrm{T}}, \mathrm{a}_{\mathrm{F}}\right)$ have a low level of confidence.

TABLE I. Number OF MESSAGES AND TIME TICKS NEEDED FOR RESOLVING THE CONFLICTS SET IN TEST 3

\begin{tabular}{|l|l|l|l|}
\hline Iteration No. & Iteration 1 & Iteration 2 & Iteration 3 \\
\hline $\begin{array}{l}\text { No. of Conflicting } \\
\text { Agents }\end{array}$ & 2 & 2 & 2 \\
\hline CR Strategy & Arbitration & Arbitration & Arbitration \\
\hline No. of Messages & 2 & 2 & 2 \\
\hline No. of Time Ticks & 2 & 2 & 2 \\
\hline
\end{tabular}

TABLE II. NUMBER OF MESSAGES AND TIME TICKS THAT ARE NEEDED FOR RESOLVING THE CONFLICTS SET IN TEST 4

\begin{tabular}{|l|l|l|l|}
\hline Iteration No. & Iteration 1 & Iteration 2 & Iteration 3 \\
\hline $\begin{array}{l}\text { No. of Conflicting } \\
\text { Agents }\end{array}$ & 2 & 2 & 2 \\
\hline CR Strategy & Negotiation & Negotiation & Negotiation \\
\hline No. of Messages & 11 & 11 & 11 \\
\hline No. of Time Ticks & 5 & 5 & 5 \\
\hline
\end{tabular}

\section{3) Other Tests}

This subsection provides other test descriptions:

Test 6: This test detects suitable strategies for resolving strong conflicts when $50 \%$ of conflicting agents $\left(a_{S}, a_{F}\right)$ have a high level of confidence and $50 \%$ of agents $\left(\mathrm{a}_{\mathrm{P}}, \mathrm{a}_{\mathrm{T}}\right)$ have a low level of confidence in a sequence of conflict as: $a_{S}, a_{P}, a_{T}, a_{F}$

Test 7: This test detects suitable strategies for resolving strong conflicts when $50 \%$ of conflicting agents $\left(\mathrm{a}_{\mathrm{P}}, \mathrm{a}_{\mathrm{T}}\right)$ have a high level of confidence and $50 \%$ of agents $\left(a_{S}, a_{F}\right)$ have a low level of confidence in a sequence of conflict as: $\mathrm{a}_{\mathrm{S}}, \mathrm{a}_{\mathrm{P}}, \mathrm{a}_{\mathrm{T}}, \mathrm{a}_{\mathrm{F}}$.

Test 8: This test detects suitable strategies for resolving strong conflicts when $50 \%$ of conflicting agents $\left(\mathrm{a}_{\mathrm{T}}, \mathrm{a}_{\mathrm{F}}\right)$ have a high level of confidence and other two $50 \%$ of agents $\left(a_{\mathrm{S}}, \mathrm{a}_{\mathrm{P}}\right)$ have a low level of confidence in a sequence of conflict: $a_{s}$, $a_{\mathrm{p}}$, $\mathrm{a}_{\mathrm{T}}, \mathrm{a}_{\mathrm{F}}$.

Test 9: This test detects suitable strategies for resolving conflicts when $25 \%$ of conflicting agents $\left(a_{S}\right)$ have a high level of confidence and $75 \%$ of conflicting agents $\left(\mathrm{a}_{\mathrm{P}}, \mathrm{a}_{\mathrm{T}}, \mathrm{a}_{\mathrm{F}}\right)$ have a low level of confidence in a sequence of conflicts: $a_{S}, a_{P}, a_{T}, a_{F}$.

Test 10: This test detects suitable strategies for resolving conflicts when $25 \%$ of conflicting agents $\left(\mathrm{a}_{\mathrm{S}}\right)$ have a high level of confidence and $75 \%$ of conflicting agents $\left(\mathrm{a}_{\mathrm{P}}, \mathrm{a}_{\mathrm{T}}, \mathrm{a}_{\mathrm{F}}\right)$ have a low level of confidence in a sequence of conflict as: $a_{\mathrm{P}}, \mathrm{a}_{\mathrm{T}}$, $\mathrm{a}_{\mathrm{S},} \mathrm{a}_{\mathrm{F}}$.

Test 11: This test detects suitable strategies for resolving conflicts when $25 \%$ of conflicting agents $\left(a_{S}\right)$ have a low level of confidence and $75 \%$ of conflicting agents $\left(a_{\mathrm{P}}, \mathrm{a}_{\mathrm{T}}, \mathrm{a}_{\mathrm{F}}\right.$ ) have a high level of confidence in a sequence of conflict as: $a_{S}$, $a_{P}$, $\mathrm{a}_{\mathrm{T}}, \mathrm{a}_{\mathrm{F}}$.

Fig. 10 shows the number of messages and time ticks needed for resolving strong conflicts when $50 \%$ of agents have high level of confidence and 50\% of agents have low level of confidence. The number of messages are around 6 to 15 and the number of time ticks around 6 to 9 . It is considered low when compare to the number of messages and time ticks generated by Test 3 .

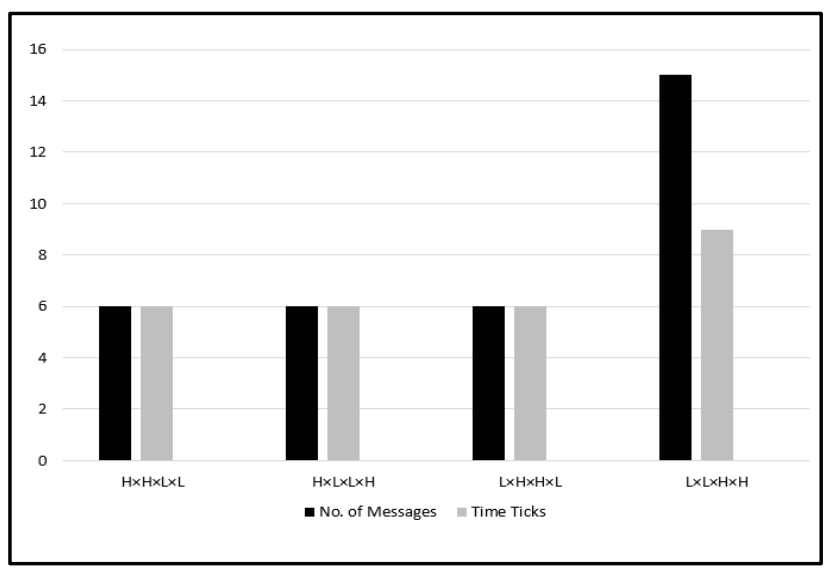

Fig. 10. The number of messages and time ticks for resolving strong conflicts among different sequence of agents when $50 \%$ of conflicting agents have high confidence and $50 \%$ of conflicting agents have low confidence.

Fig. 11 shows the number of messages and time ticks for resolving weak conflicts among four agents that have an equal confidence level. Note that ConfRSSM ignores weak conflicts among low confidence agents, and the number of messages and time ticks equals zero.

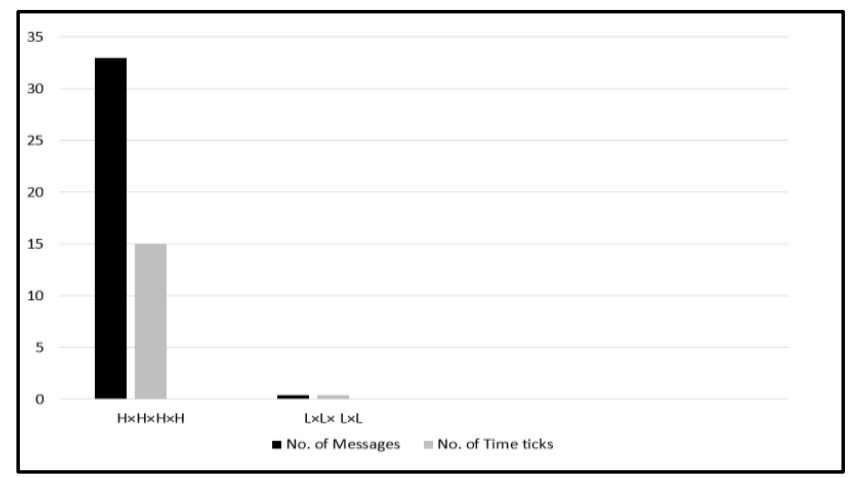

Fig. 11. The number of messages and time ticks needed for resolving conflicts among agents that have the same confidence level when the conflicts are weak. 
Fig. 12 shows the number of messages and time ticks needed for resolving conflicts when a $75 \%$ of conflicting agents have a low level confidence, and the other $25 \%$ of conflicting agents have high confidence level, taking into consideration a multiple conflicts sequences. The result clearly shows that there is a decrease in the number of messages and time ticks in all conflict states.

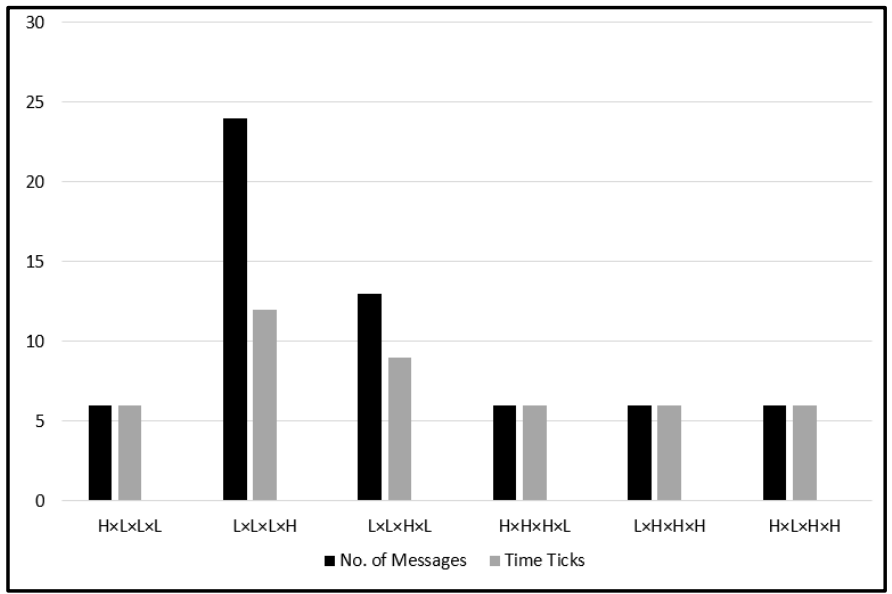

Fig. 12. The number of messages and time ticks for resolving strong conflicts among agents when $75 \%$ of conflicting agents have an equal confidence level and $25 \%$ have an opposite confidence level.

Test 12: This test detects the strategies for resolving conflicts when $25 \%$ of conflicting agents $\left(\mathrm{a}_{\mathrm{s}}\right)$ have a low level of confidence and $75 \%$ of conflicting agents $\left(\mathrm{a}_{\mathrm{P}}, \mathrm{a}_{\mathrm{T}}, \mathrm{a}_{\mathrm{F}}\right)$ have a high level of confidence in a sequence of conflict: $a_{P}, a_{S}, a_{T}, a_{F}$.

Test 13: This test determines the conflict resolution strategies when all conflicting agents have a high confidence level and the conflict among them is weak.

Test 14: This test determines the conflict resolution strategies when all conflicting agents have a low confidence level and the conflict among them is weak.
Test 15: This test detects suitable strategies for resolving conflicts when two of the conflicting agents $\left(\mathrm{a}_{\mathrm{s}}, \mathrm{a}_{\mathrm{p}}\right)$ have a high level of confidence and other two agents $\left(\mathrm{a}_{\mathrm{T}}, \mathrm{a}_{\mathrm{F}}\right)$ have a low level of confidence and the conflicts are weak in a sequence of conflicts: $\mathrm{a}_{\mathrm{S}}, \mathrm{a}_{\mathrm{P}}, \mathrm{a}_{\mathrm{T}}, \mathrm{a}_{\mathrm{F}}$.

Test 16: This test detects suitable strategies for resolving conflicts when one of conflicting agents $\left(a_{s}\right)$ have a high level of confidence and other three agents $\left(\mathrm{a}_{\mathrm{P}}, \mathrm{a}_{\mathrm{T}}, \mathrm{a}_{\mathrm{F}}\right)$ have a low level of confidence and conflicts are weak in a sequence of conflicts: $\mathrm{a}_{\mathrm{S}}, \mathrm{a}_{\mathrm{P}}, \mathrm{a}_{\mathrm{T}}, \mathrm{a}_{\mathrm{F}}$.

Fig. 13 shows the number of messages and time ticks for resolving the weak conflicts when 50\% agents have high level of confidence and other $50 \%$ of agents have low level of confidence. The number of messages and time ticks are lower because conflict resolution is equipped with ConfRSSM. The number of messages are around 13 to 15 and the number of time ticks around 7 to 9 .

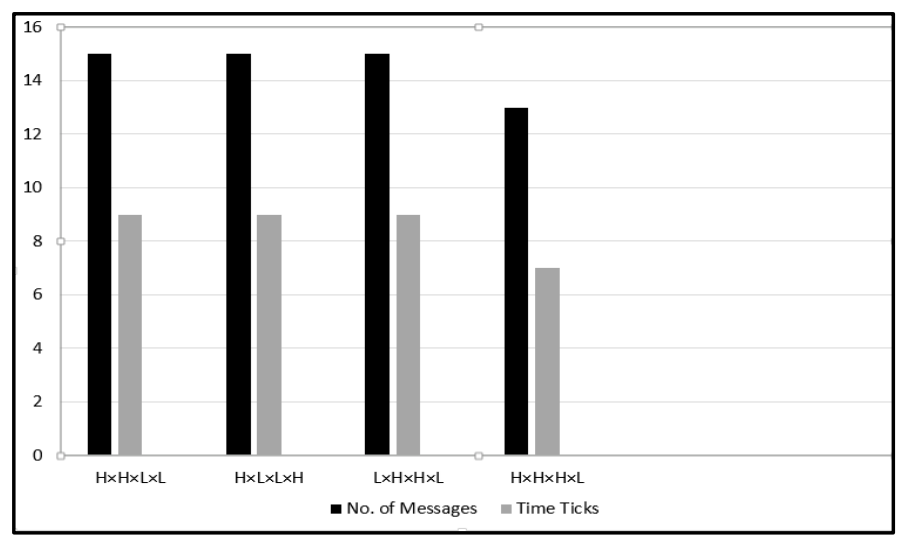

Fig. 13. The number of messages and time ticks for resolving strong conflicts among different sequence of agents when $50 \%$ of conflicting agents have high confidence and $50 \%$ of conflicting agents have low confidence.

Results of simulation are summarized in Table 3.

TABLE III. SiMULATION RESULTS FOR SELECTED TEST CASES

\begin{tabular}{|l|l|l|l|l|}
\hline $\begin{array}{l}\text { Confidence Level of } \\
\text { Conflicting Agents }\end{array}$ & $\begin{array}{l}\text { Conflict } \\
\text { State }\end{array}$ & Conflict Resolution Strategy & $\begin{array}{l}\text { No. of } \\
\text { Messages }\end{array}$ & $\begin{array}{l}\text { No. of Time } \\
\text { Ticks }\end{array}$ \\
\hline HLC, LLC, LLC, HLC & Strong & Forcing, Forcing, Arbitration & 6 & 6 \\
\hline LLC, HLC, HLC, LLC & Strong & Forcing, Arbitration, Forcing & 6 & 6 \\
\hline LLC, LLC, HLC, HLC & Strong & Negotiation,Forcing, Arbitration & 15 & 6 \\
\hline HLC, LLC, LLC, LLC & Strong & Forcing, Forcing, Forcing, & 6 & 6 \\
\hline LLC, LLC, LLC, HLC & Strong & Negotiation, Negotiation, Forcing & 24 & 6 \\
\hline LLC, LLC, HLC, HLC & Strong & Negotiation, Negotiation, Forcing & 15 & 9 \\
\hline LLC, HLC, HLC, HLC & Strong & Forcing, Arbitration, Arbitration & 6 & 9 \\
\hline HLC, HLC, HLC, LLC & Strong & Arbitration, Arbitration, Forcing & 6 & 6 \\
\hline HLC, LLC, HLC, HLC & Strong & Forcing, Arbitration, Arbitration & 6 & 6 \\
\hline HLC, HLC, LLC, LLC & Weak & Negotiation, Negotiation, Negotiation & 33 & 6 \\
\hline LLC, LLC, LLC, LLC & Weak & Ignoring, Ignoring, Ignoring & 0 & 15 \\
\hline HLC, HLC, LLC, LLC & Weak & Negotiation, Submitting, Submitting & 15 & 0 \\
\hline
\end{tabular}




\begin{tabular}{|l|l|l|l|l|}
\hline LLC, HLC, HLC, LLC & Weak & Submitting, Negotiation, Submitting & 15 & 9 \\
\hline LLC, LLC, HLC, HLC & Weak & Ignoring, Submitting, Negotiation & 13 & 9 \\
\hline HLC, LLC, LLC, LLC & Weak & Submitting, Submitting, Submitting & 6 & 7 \\
\hline LLC, LLC, LLC, HLC & Weak & Ignoring, Ignoring, Submitting & 2 & 6 \\
\hline LLC, LLC, HLC, HLC & Weak & Ignoring, Submitting, Submitting & 4 & 2 \\
\hline LLC, HLC, HLC, HLC & Weak & Submitting, Negotiation, Negotiation & 24 & 4 \\
\hline HLC, HLC, HLC, LLC & Weak & Negotiation, Negotiation, Submitting & 24 & 12 \\
\hline HLC, LLC, HLC, HLC & Weak & Submitting, Negotiation, Negotiation & 24 & 12 \\
\hline
\end{tabular}

\section{Result DisCUSSION}

The messages and time required in weak conflict cases are low (may reduce to zero) as the result of using Ignoring strategy. This means that weak conflcits are totally ignored when the confidence level of conflciting agents is low (e.g. Test 10). There is a clear dcreasing in the number of messages and time ticks for resolving a strong and weak conflicts among multiple conflicts sequence of agents that have a diffrent confidence levels. Also, results show that the number of messages and time ticks for resolving conflicts using Negotiation considered high when agents are using more one proposals. This is critical for multi-agent systems. The high messages in Test 3; Tests 5-6; Tests 9-10; and Tests 15-16 are due to the application of Negotiation strategy. The strategy needs high message number to process as compared to other conflict resolution strategies. There is an obvious decrease in the number of messages and time ticks for resolving a strong and weak conflicts among multiple conflict sequence among agents that have a different confidence level. The number of messages are high (around 9 to 24) in all conflict states that contains conflict between two high confidence agents. While in conflict states that includes two low confidence conflicting agents, the number of messages low (around 2 to 6 ).

\section{CONCLUSION}

Conflicts are likely to be the most critical parameter manifested through agent communication in a distributed multi-agent system. One of the most difficult aspects of the current interest in agent system is selecting an appropriate conflict resolution strategy. Classifying conflict states facilitate the selection of an optimal strategy to resolve conflicts in every conflict situation. Since there is no better strategy suitable for all conflict situations, agent-based systems would benefit from the multiple resolution strategies to resolve unanticipated conflicts. This research attempts to prove the significance of giving software agents the ability to select an appropriate strategy in different conflict states depending on the conflict strengths and confidence levels of the conflicting agents. We presented a novel method to guide strategic decision-making for conflict resolution, and adopted four basic strategies (i.e. Negotiation, Arbitration, Ignoring, and Submitting). In the simulation part, various senarios were tested with different conflicts among four agents running with the proposed ConfRSSM framework. As expected, using Ignoring, Forcing and Submitting strategies enhanced the conflict resolution performance by decreasing the number of messages and time ticks. Results show ConfRSSM reduces the number of messages and time ticks and thus improving the conflict resolution process. Further analysis shows that some unimportant conflict states can be ignored, which increases the efficiency of the entire conflict resolution process.

\section{REFERENCES}

[1] Nguyen, N. T. (2002). Consensus System for Solving Conflicts in Distributed Systems. Journal of Information Sciences, 147(1-4), 91-122.

[2] Tessier, C., Chaudron, L., Muller, H.J. (2000). Conflict Agents, Conflict Management in Multi Agent System, 1. Springer, Heidelberg.

[3] Liu, T. H., Goel, A., Martin, C. E. \& Barber, K. S. (1998). Classification and Representation of Conflict in Multi-agents Systems. Technical Report TR98-UT-LIPSAGENTS-01, The Laboratory for Intelligent Processes and Systems, University of Texas at Austin.

[4] M"uller, H. J. \& Dieng, R. (2000). Computational Conflicts- Conflict Modeling for Distributed Artificial Intelligent Systems. Springer Verlag Publishers.

[5] Barber K. S., Kim J., (2001). Belief Revision Process Based on Trust: Simulation Experiments, In Proceedings of Autonomous Agents Workshop on Deception, Fraud, and Trust in Agent Societies.

[6] Decker, K. S., \& Lesser, V. R. (1995). Environment Centered Analysis and Design of Coordination Mechanisms (Doctoral dissertation, University of Massachusetts at Amherst).

[7] Wang, Y., Mellon, C. \& Singh, M. P. (2010). Evidence-Based Trust A Mathematical Model Geared for Multiagent Systems, ACM Transaction on Autonomous and Adaptive Systems (TAAS), 5(4), (pp.1-28).

[8] Yu, B. \& Singh, M. P. (2002). Distributed Reputation Management for Electronic Commerce. Computational Intelligence, 18(4), (pp. 5-549).

[9] Yu, Han, Zhiqi Shen, C. Y. R. I. L. Leung, Chunyan Miao \& VICTOR R. Lesser. (2013). A Survey of Multi-agent Trust Management Systems. Access, IEEE 1, (pp. 35-50).

[10] Sen, S. \& Sajja, N. (2002). Robustness of Reputation-based Trust: Booblean Case. Proceedings of the First International Joint Conference on Autonomous Agents and Multiagent Systems, (pp. 288-293), ACM Press.

[11] Wagner T., Shapiro, J., Xuan, P., Lesser, V., (2007). Multi-level Conflict in Multi-Agent Systems. Proceeding of AAAI Workshop on Negotiation in Multi-Agent Systems.

[12] Alshabi, W., Ramaswamy, S., Itmi, M., \& Abdulrab, H. (2007). Coordination, Cooperation and Conflict Resolution in Multi-agent Systems. In Innovations and Advanced Techniques in Computer and Information Sciences and Engineering (pp. 495-500). Springer Netherlands.

[13] Adler, M., Durfee, E., Huhns, M., Punch, W., \& Simoudis, E. (1992). AAAI workshop on cooperation among heterogeneous intelligent agents. AI magazine, 13(2), 39.

[14] Alonso, E., d'Inverno, M., Kudenko, D., Luck, M. \& Noble, J. (2001). Learning in Multi-agent Systems. Result of a Panel Discussion, In: Third Workshop of the UK's Special Interest Group on Multi-agent Systems.

[15] Boff, E., Vicari, R. M., \& Fagundes, M. S. (2008). Using a Probabilistic Agent to Support Learning in Small Groups. In The 22nd European Conference on Modeling and Simulation, ECMS.

[16] Ghusoon Salim Basheer, Mohd Sharifuddin Ahmad, Alicia Y.C. Tang, Sabine Graf, "Certainty, Trust and Evidence: Towards an Integrative 
Model of Confidence in Multi-agent Systems", Computers in Human Behavior, Volume 45, Elsevier, April 2015, Pages 307-315, ISSN: 0747-5632, (Impact Factor 2.273), DOI: 10.1016/j.chb.2014.12.030.

[17] Ghusoon Salim Basheer, Mohd Sharifuddin Ahmad, Alicia Y.C. Tang, Azhana Ahmad, and Mohd. Zaliman Yussof, "A Novel Conflict Resolution Strategy in Multi-agent Systems: Concept and Model", Advanced Approaches to Intelligent Information and Database Systems, study in computational intelligent, Lecture Note in Artificial Intelligence, Volume 551, pp. 35-45, 2014.

[18] Ghusoon Salim Basheer, Mohd Sharifuddin Ahmad, Alicia Y. C. Tang, "A Conflict Classification and Resolution Modeling in Multi-agent Systems", Encyclopedia of Information Science and Technology (3rd Ed.), DOI: 10.4018/978-1-4666-5888-2.ch685.

[19] Ghusoon Salim Basheer, Mohd Sharifuddin Ahmad, and Alicia Y.C. Tang, A Framework for Conflict Resolution in Multi-agent Systems, 5th International Conference on Computational Collective Intelligence Technologies and Applications (ICCCI 2013), 11-13 September 2013, Craiova, Romania. Lecture Note in Computer Science, Volume 8083, pp. 195-204, 2013.
[20] Ghusoon Salim Basheer, Mohd Sharifuddin Ahmad, Alicia Y. C. Tang, A Conceptual Multi-agent Framework using Ant Colony Optimization and Fuzzy Algorithms for Learning Style Detection, 5th Asian Conference, ACIIDS, Kuala Lumpur, Malaysia, March 18 - 20, 2013, Proceedings, Part II: Intelligent Information and Database Systems, Lecture Notes in Artificial Intelligence (LNAI), Vol. 7803, pp. 549-558, 2013.

[21] Ghusoon Salim Basheer, Alicia Y.C. Tang, Mohd Sharifuddin Ahmad, Designing Teachers' Observation Questionnaire based on Curry's Onion Model for Students' Learning Styles Detection, TEM Journal. Volume 5, Issue 4, Pages 515-521, ISSN 2217-8309, DOI: 10.18421/TEM54-16.

[22] Fleming, N., (2006). I'm different; Not Dumb, Modes of Presentation [V.A.R.K.] in the Tertiary Classroom.

[23] Fleming, N. (2001). VARK a Guide to Learning Style.

[24] Alicia Y.C. Tang and Ghusoon Salim Basheer, A Conflict Resolution Strategy Selection Method (ConfRSSM) in Multi-Agent Systems, International Journal of Advanced Computer Science and Applications (IJACSA), Vol 8, Issue 5, June 2017, pp. 398-404. 